\title{
Acceptance of COVID-19 Vaccination and its Related Factors Among HIV/AIDS Patients in Guangxi, China: A Cross-Sectional Survey.
}

Jinming Su

Guangxi Medical University

Zhenwei Jia

Guangxi Medical University

Fengxiang Qin

Guangxi Medical University

Rongfeng Chen

Guangxi Medical University

Yuting Wu

Guangxi Medical University

Beibei Lu

Guangxi Medical University

Chunlin Lan

Guangxi Medical University

Tongxue Qin

Guangxi Medical University

Yinlu Liao

Guangxi Medical University

Minjuan Shi

Guangxi Medical University

Yanyan Liao

Guangxi Medical University

Peijiang Pan

Guangxi Medical University

Li Ye

Guangxi Medical University

Junjun Jiang

Guangxi Medical University

Hao Liang ( $\nabla$ lianghao@gxmu.edu.cn )

Guangxi Medical University 


\section{Research Article}

Keywords: HIV/AIDS, COVID-19, SARS-CoV-2, Vaccine, Acceptance

Posted Date: January 3rd, 2022

DOI: https://doi.org/10.21203/rs.3.rs-1109933/v1

License: (c) (i) This work is licensed under a Creative Commons Attribution 4.0 International License. Read Full License 


\section{Abstract \\ Background}

Vaccination is the most effective approach against the coronavirus disease 2019 (COVID-2019) pandemic. This study aimed to investigate acceptance and the predominant influential factors of COVID19 vaccination among people living with human immunodeficiency virus (HIV)/acquired immunodeficiency syndrome (AIDS).

\section{Methods}

A cross-sectional survey was carried out in five cities in Guangxi from 7 May to 1 June 2021. Questionnaires on the acceptance of COVID-19 vaccination and the influential factors were conducted among HIV/AIDS patients recruited by random cluster sampling. We performed univariate and multivariate logistic regression analysis to identify factors associated with acceptance of COVID-19 vaccination among HIV/AIDS patients.

\section{Results}

Of all the participants ( $n=903), 72.9 \%(n=658)$ were willing to accept the COVID-19 vaccine and there was no statistically significant difference between $\mathrm{CD} 4^{+} \mathrm{T}$ cell count and willingness to vaccinate using stratified analysis $(P>0.05)$. The main reason for willingness to accept the COVID-19 vaccine was fear of severe acute respiratory syndrome coronavirus 2 (SARS-CoV-2) infection $(76.0 \%)$, whereas patients who were reluctant to receive the vaccine were mainly concerned about the safety of the vaccine $(54.7 \%)$ and whether it would impact anti-retroviral therapy(ART) efficacy (50.6\%). The most significant factors influencing vaccination were concerns that the vaccine was unsafe in HIV patients $(O R=0.082,95 \% \mathrm{Cl}=$ 0.024-0.282) and that it would be less effective in preventing SARS-CoV-2 infection in HIV patients (OR = $0.093,95 \% \mathrm{Cl}=0.030-0.287)$. Other factors associated with acceptance of the COVID-19 vaccine included Zhuang ethnicity $(\mathrm{OR}=1.653,95 \% \mathrm{Cl}=1.109-2.465)$, the highest education level of middle school and high school or above (OR=1.747, 95\%Cl=1.170-2.608; $\mathrm{OR}=2.492,95 \% \mathrm{Cl}=1.326-4.682)$, unknown vaccination type $(\mathrm{OR}=0.487,95 \% \mathrm{Cl}=0.305-0.776)$ and little vaccine influence on $A R T$ efficacy $(\mathrm{OR}=2.889$, $95 \% \mathrm{Cl}=1.378-6.059)$.

\section{Conclusions}

Acceptance of the COVID-19 vaccination is high among HIV/AIDS patients, although some patients refused vaccination because of vaccine safety and influence on ART efficacy. More research is needed to investigate the impact of the COVID-19 vaccine on the efficacy of ART and to evaluate its effectiveness in 
preventing SARS-CoV-2 infection in HIV patients so that concerns about COVID-19 vaccination issues can be addressed in HIV patients.

\section{Background}

The coronavirus disease 2019 (COVID-19) pandemic has caused an enormous social and economic burden throughout the world, with more than 238 million COVID-19 confirmed cases and over 4.8 million deaths globally as of 9 October 2021[1]. Currently, specific prophylactic drugs targeting severe acute respiratory syndrome coronavirus 2 (SARS-CoV-2) are under development[2] but vaccination is the most cost-effective approach to slow virus spread and control this outbreak[3]. To date, more than 6.3 billion doses of vaccines have been administered worldwide, of which nearly 2.2 billion have been used in China[1]. It is well known that most people are immunized against the virus after vaccination, which reduces the risk of virus infection and the severity and mortality of the disease. The effectiveness of the COVID-19 vaccine has been validated by clinical trials and real-world studies, both of which have shown the vaccine to be effective for fighting SARS-CoV-2 and for significantly improving multiple disease outcomes in COVID-19[4, 5]. This would create a broad population immune barrier and thus control the COVID-19 pandemic[6]. There is no doubt that vaccination is an essential measure to restore a full economic life.

During the COVID-19 pandemic, there were many studies on the high risk factors for COVID-19, such as elderly, hypertension, overweight, diabetes and cardiovascular disease[7, 8]. Also, people with human immunodeficiency virus (HIV)/acquired immunodeficiency syndrome (AIDS) should not be ignored because they will have more severe symptoms once they are infected with SARS-CoV-2. By the end of 2020 , roughly 37.7 million people were reported to be living with HIV around the world[9]. As of October 2019 there were about 958 thousand SARS-CoV-2 infected HIV/AIDS patients in China and although the overall epidemic continues to be at a low level AIDS is still the most infectious disease in terms of reported deaths[10]. This population is characterized by a persistent decrease in the CD $4^{+} T$ cell count and a dysregulated immune system[11]. Therefore, some studies concluded that HIV/AIDS patients may suffer more severity or mortality of COVID-19 combined with previous chronic disease[12, 13]. Obviously, we have to face the daunting challenge and crisis for HIV/AIDS patients, which may create a more intense medical burden due to the COVID-19 pandemic.

A South African cohort study reported that people infected with HIV, particularly those not on antiretroviral therapy (ART), were at high risk of COVID-19 in-hospital mortality and would benefit from vaccine prioritization[14]. A systematic review also showed that HIV-positive persons had a significantly higher risk of SARS-CoV-2 infection and mortality than HIV-negative individuals, suggesting that people with HIV need priority consideration for the COVID-19 vaccine[15]. Although some studies have suggested that HIV/AIDS patients are at high risk of SARS-CoV-2 infection and should be given the COVID-19 vaccine as a priority, there are still insufficient large-scale data on the safety and efficacy of the COVID-19 vaccine for HIV-infected patients. How to choose COVID-19 vaccines for HIV-infected patients has been a 
hot topic of concern. At the same time, their willingness to be vaccinated with these vaccines is an important issue to be considered.

In this study, we selected HIV/AIDS patients in Guangxi to investigate their COVID-19 knowledge, attitude, acceptance and influencing factors for COVID-19 vaccination. First, the AIDS epidemic is still very grim in Guangxi, with a high prevalence[16]. Second, Guangxi is located in western China, bordering Southeast Asia, with a unique geographical environment and population diversity that may be very susceptible to COVID-19/HIV cross-border transmission and dual infection[17]. Furthermore, there were no data on acceptance of the COVID-19 vaccine or the influencing factors for people living with HIV in China currently. We aimed to investigate acceptance and the factors associated with COVID-19 vaccination among HIV/AIDS patients in Guangxi in order to provide behavioural intervention and vaccination strategies to tackle the COVID-19 pandemic among this unique population.

\section{Methods}

\section{Study design, subjects and sampling}

A cross-sectional survey was conducted in the form of face-to-face interviews across five cities in Guangxi (Chongzuo, Guigang, Laibing, Qinzhou, Yulin) from 7 May to 1 June 2021. Participants were aged 18 years and above, residing in Guangxi, confirmed as HIV-1 positive using a stratified random sampling method and with no confirmed COVID-19 status. In total, 1000 respondents were randomly selected and 988 questionnaires were collected (98.8\%), the final sample consisted of 903 respondents (91.4\%) after quality control and an initial check to exclude 85 incomplete and invalid questionnaires. All participants signed informed consent forms in this survey. The study was approved by the Ethics and Human Subjects Committee (EHSC) of Guangxi Medical University[No.20210153].

\section{Questionnaires and data management}

The self-designed questionnaire was based on previous research evaluating the public's vaccination willingness $[18,19]$ and aimed at obtaining information on the acceptability of the COVID-19 vaccine among HIV/AIDS patients as an effective strategy against the coronavirus. The survey consisted of several subsections: demographic characteristics and health status, general status of HIV infection, knowledge of COVID-19 and its vaccine, and attitude towards COVID-19 vaccination with HIV infection.

All respondents were asked about individual characteristics and health status, such as age, gender, marital status, education level, occupation, ethnicity, height, weight and history of severe chronic disease. The general status of HIV infection was collected using the China Information System for Diseases Control and Prevention, including the pathway of HIV transmission and infection, $\mathrm{CD} 4^{+} \mathrm{T}$ cell count, infectious time, ART status and adverse effects of ART.

To acquire participants' knowledge of COVID-19 and its vaccination, the following four questions were asked: "What are the main ways to learn about COVID-19?", "What are the available vaccines in China?", 
"What do you think is the most appropriate age to receive the COVID-19 vaccine?" and "Which type of occupation is preferential for COVID-19 vaccination?".

For perception of COVID-19 vaccination with HIV infection, six questions were asked on factors impacting COVID-19 vaccination willingness among the participants: respondents' knowledge of China's vaccine policy and types was assessed with "Do you know which COVID-19 vaccine is free in China?" and "Which type of COVID-19 vaccine is better?", perceived vaccine validity and safety was evaluated with "Do you think COVID-19 vaccination is helpful to control the pandemic?" and "Do you think COVID-19 vaccination is safe?", and susceptibility factors between COVID-19 and HIV infection were probed with "Is COVID-19 vaccination effective for HIV/AIDS patients?" and "Do you think COVID-19 vaccination affects ART efficacy?".

The aim of the last section was to investigate attitude towards COVID-19 vaccination. For example, the 'willing to be vaccinated' (WTV) group were asked "Which COVID-19 vaccine would you like to choose", "Why do you want to receive the COVID-19 vaccine?", "Would you still want to receive the COVID-19 vaccine if our country charged for it?" and "Do you still wear a mask after vaccination?", whereas the 'unwilling to be vaccinated' (non-WTV) group were asked "Why don't you want to receive the COVID-19 vaccine?", "Would you receive the COVID-19 vaccine if it was freely accessible in China?" and "Would you accept the COVID-19 vaccine if most people have already been vaccinated in China?".

\section{Statistical analysis}

For analysis, we defined respondents who chose "Yes" to the question "Would you accept the COVID-19 vaccine during this pandemic in China" as the WTV group and those who chose "No" as the non-WTV group. All data were entered into EpiData software (EpiData 3.1 for Windows; The EpiData Association, Odense, Denmark) and analysed using SPSS for Windows Version 23.0 (SPSS, Chicago, IL, USA).

Descriptive statistics were generated for each of the variables, corresponding to specific questions in the survey, including: general characteristics, knowledge of and attitude to COVID-19 and its vaccine, and reasons for accepting or refusing COVID-19 vaccination. We performed univariate and multivariate logistic regression analysis to identify factors associated with the acceptability of COVID-19 vaccinations among HIV/AIDS patients. Variables that showed statistically significant associations $(P<0.05)$ with willingness to be vaccinated were included in analyses and the odds ratio (OR), adjusted OR (aOR) and $95 \%$ confidence interval $(\mathrm{Cl})$ were calculated. All statistical tests were two sided with a significance level of $P<0.05$.

\section{Results}

\section{Demographic characteristics and HIV status}

Of the 988 subjects interviewed, 903 completed the questionnaires (response rate $=91.4 \%$ ) and 658 (72.9\%) of these were willing to accept the COVID-19 vaccine in order to prevent SARS-CoV-2. As shown in Table $1,64 \%$ of respondents were male, $47.6 \%$ were $41-59$ years old, $62.5 \%$ were of Han ethnicity, $63.7 \%$ 
were farmers and $63.1 \%$ were married. Regarding education level, $36.8 \%$ were educated to primary school level or below and $47.0 \%$ to middle school level. With regard to health status, $67.4 \%$ had a normal body mass index (BMI) and $90.6 \%$ thought they did not have a serious chronic illness. In terms of HIV status, $46.3 \%$ had $C D 4^{+}$T cell counts of $200-500$ cells $/ \mu L, 95.5 \%$ were infected through sexual transmission, $56.4 \%$ had been infectious for more than 5 years, $92.2 \%$ had received ART and $10.6 \%$ had some side effects of ART. The results of stratified analysis (Table 2 ) show no statistically significant difference in the effect of different $\mathrm{CD} 4^{+} T$ cell levels on willingness to be vaccinated $(P>0.05)$.

\section{Knowledge of and attitude to COVID-19 vaccination}

Knowledge of COVID-19 and its vaccination is shown in Table 3. Most respondents learn about COVID-19 through their phone $(68.4 \%)$ and the TV $(59.4 \%)$. Nearly half of subjects $(44.7 \%)$ reported that inactivated vaccine is available in China whereas $45.4 \%$ hold the views of unknown vaccine. In terms of the appropriate age and occupation for COVID-19 vaccination, they though the age between 18 and 40 years $(66.7 \%)$ and the medical personnel $(74.3 \%)$ should be preferred.

When probing the reasons for participants' willingness or unwillingness to be vaccinated (Tables 4 and 5), we found that the main reason for vaccination was fear of SARS-CoV-2 infection (76.0\%), with the second major reason being because their relatives and friends were willing to accept the vaccines. The main reason against vaccination was the adverse effects (54.7\%), with the second major reason being that the vaccine will affect ART efficacy (50.6\%). The WTV group showed greater preference for inactivated vaccine $(69.1 \%)$ and declared that they would still want to receive the COVID-19 vaccine even if it was charged for in the future (69.5\%). The non-WTV group refused to accept the vaccine even though it is freely accessible in China (67.3\%) and would still refuse even if most people were vaccinated in the future (62.0\%). In addition, on investigating behaviour towards COVID-19 vaccination, the results showed that most of the WTV group still insisted on wearing a mask after vaccination for better protection against COVID-19 (89.2\%).

\section{Factors associated with acceptability of COVID-19 vaccination among HIV/AIDS patients}

A comparison of attitude towards COVID-19 vaccination with HIV infection is shown in Table 6 and Supplementary Additional file 1. Compared to the non-WTV group, more respondents in the WTV group knew that the COVID-19 vaccine is free in China at present $(93.8 \%$ versus $86.5 \% ; P<0.05)$, and thought that the vaccination is helpful or largely helpful in controlling the grim pandemic $(86.0 \%$ versus $59.6 \%$; $P<$ 0.05). Furthermore, in comparison with the non-WTV group, more than half of the WTV group showed higher preference for domestic vaccines $(74.8 \%$ versus $44.9 \% ; P<0.05)$. In terms of important perceptions on vaccination in relation to HIV, more of the WTV group believed that COVID-19 vaccination did not affect ART efficacy compared with the non-WTV group (34.7\% versus $11.8 \% ; P<0.05)$. On further analysis, multivariable logistic regression identified six influencing factors associated with vaccine acceptability among HIV/AIDS patients (Table 6): ethnicity (Zhuang versus Han: OR $=1.653,95 \% \mathrm{Cl}=$ 1.109-2.465), highest level of education (middle school versus primary school or below: $\mathrm{OR}=1.747$, 
$95 \% \mathrm{Cl}=1.170-2.608$; high school and above versus primary school or below: $\mathrm{OR}=2.492,95 \% \mathrm{Cl}=$ $1.326-4.682$ ), vaccination safety (No versus Yes: $\mathrm{OR}=0.082,95 \% \mathrm{Cl}=0.024-0.282$; Unknown versus Yes: $\mathrm{OR}=0.381,95 \% \mathrm{Cl}=0.233-0.624$ ), type of vaccine (uncertain/unknown vaccine versus domestic vaccine: $\mathrm{OR}=0.487,95 \% \mathrm{Cl}=0.305-0.776)$, effectiveness of COVID-19 vaccination in HIV/AIDS patients (worse versus better: $\mathrm{OR}=0.093,95 \% \mathrm{Cl}=0.030-0.287$ ) and whether COVID-19 vaccination affects ART efficacy (No versus Yes: $\mathrm{OR}=2.889,95 \% \mathrm{Cl}=1.378-6.059$ ). Other factors that were not statistically significant are shown in an additional file in detail [see Additional file 1].

\section{Discussion}

Our investigation is the first to investigate the acceptance of COVID-19 vaccines and its related factors among HIV/AIDS patients in China. This survey showed a lower rate (72.9\%) of COVID-19 vaccination acceptance among HIV-infected patients compared to the general population (91.3\%) in China[18], although it is higher than in other countries such as Russia (54.85\%), France (58.89\%) and Sweden (65.23\%), according to global research[20]. There were many studies showing that HIV/AIDS patients were at high risk of COVID-19 in severe manifestations, hospitalization and hospital mortality relative to HIV-negative persons[12, 14, 21]. Furthermore, many countries reported that the delivery of HIV healthcare services has been disrupted by COVID-19 and the consequences may increase morbidity and mortality in HIV-infected patients[22, 23]. South Africa is bearing the brunt of COVID-19 as it has the highest burden of AIDS and the World Health Organization estimated more than 500,000 extra deaths from HIV in the coming year[24]. Accordingly, many countries have identified HIV-infected patients as their priority vaccination cohort $[15,22]$. Investigation into acceptance and the factors influencing vaccination for HIVinfected patients is urgent and essential but it would be more feasible and effective to implement an immunization programme among this unique population in China or even worldwide in the future.

As shown in this research, we found that most patients (68.4\%) learned about the COVID-19 epidemic but a substantial proportion of respondents $(45.4 \%)$ had little knowledge of the vaccine. The WTV group possessed better knowledge about COVID-19 vaccine policy in China, such as the free vaccine policy, compared to the non-WTV group. Furthermore, most participants preferred the inactivated vaccine, mainly because the inactivated vaccine technology is more traditional and mature $[25,26]$ and many researchers have verified that this vaccine has high efficacy against coronavirus[5, 27]. Moreover, most of the WTV group have enough positive awareness to be willing to pay for the vaccine if it was charged for in the future, which may be due to the strong implementation of policies and services for the promotion of COVID-19 vaccination in China[28]. However, some in the non-WTV group expressed that they would refuse vaccination even if there was free access without epidemic restrictions and also if a vast number of people were vaccinated in the future. Therefore, it is urgent to raise awareness of the relationship between the vaccine and AIDS in order to change their mindset.

The further analysis results revealed that vaccine safety is a strong factor affecting vaccine acceptance, which is in line with previous studies on concerns of the public[29, 30]. Many real-world studies have confirmed that COVID-19 vaccines are safe and effective around the world, including inactivated vaccine 
and mRNA vaccine[27, 31]. The amount of research above should, in theory, give great confidence to the public on vaccine safety. However, the survey reflected that HIV/AIDS patients are still concerned about the side effects and effectiveness of COVID-19 vaccine on themselves, they should be made aware that none of the COVID-19 vaccines were prepared using an attenuated vaccine strategy, which is designed to lose pathogenicity while retaining antigenicity, and therefore the vaccines may not be suitable for people with an impaired immune system[32, 33]. The effect of vaccines on ART efficacy is a great focus for HIVinfected patients: so far, no specific drug to cure AIDS has been developed and although ART is the most effective treatment available, patients still may be immunocompromised or immunosuppressed[34]. A multicentre study found that HIV/AIDS patients receiving tenofovir disoproxil fumarate (TDF)/emtricitabine (FTC) had a lower risk for COVID-19 compared with other ART regimens[35], and a South African study had similar observations[36]. In contrast, several studies reported no significance in the relationship between ART regimens and COVID-19 severity in HIV-infected patients[37, 38], although the findings are not yet conclusive, and some domestic experts recommended that HIV-infected patients on long-term antiviral therapy with a well-controlled HIV viral load and no vaccination contraindications should receive inactivated vaccine as soon as possible[39].

In addition, this study showed that the higher the education level, the higher the willingness to vaccinate, which was consistent with previous results among the general population[20, 40]. However, HIV/AIDS patients with lower education accounted for the majority in Guangxi[41], so it is necessary to strengthen knowledge of the COVID-19 vaccine among this population. Furthermore, a cohort study found that lower $\mathrm{CD} 4^{+} \mathrm{T}$ cell counts were associated with COVID-19 mortality[36], however, other studies found no correlation between CD $4^{+} T$ cell count or HIV viral load and COVID-19 outcomes[37, 42], and our results also showed that $C D 4^{+} T$ cell count was not statistically significant for acceptance of COVID-19 vaccines by stratified and multivariate analysis. From the research, our results showed no significant difference in gender and age among HIV/AIDS related to vaccine acceptance, which is inconsistent with some studies $[7,18]$ and may be attributed to the sample size of the survey.

Several limitations should be taken into account in this study. First, our survey used convenience sampling, which may lead to selection bias in some results. Second, we found no HIV-infected patients who had received the vaccine before this investigation and we had little knowledge on the side effects of the vaccine or ART efficacy among HIV-infected patients, thus we need to learn more about the postvaccination status as a next step. We will conduct follow-up research on vaccinated patients, including the side effects of vaccination and alterations in immune function, such as $C D 4^{+} T$ cell count and viral load, in order to provide some reference basis for future vaccine strategies.

\section{Conclusions}

In summary, the study indicates a high COVID-19 vaccination acceptance rate among HIV/AIDS patients. Vaccine safety and effectiveness in HIV-infected patients were identified as important factors in vaccine acceptance, which can be used to design a programme to promote vaccination among this unique population. Moreover, some patients still refused vaccination, mainly because of concerns about vaccine 
safety and influences on ART efficacy. Therefore, educational knowledge about AIDS and the vaccine should be strengthened and popularized, including the necessity for vaccine protection against SARSCoV-2, which can improve awareness to make better choices about vaccines and achieve the goal of herd immunity. HIV/AIDS patients should insist on preventive measures, whether vaccinated or not; at the same time, more attention should be paid to treatment services provision for HIV-infected patients in order to avoid increased morbidity and mortality related to HIV during the COVID-19 pandemic.

\section{Abbreviations}

COVID-19: Coronavirus disease 2019; HIV: People living with human immunodeficiency virus; AIDS: Acquired immunodeficiency syndrome; SARS-CoV-2: Severe acute respiratory syndrome coronavirus 2; ART: Anti-retroviral therapy; OR: Odds ratio; aOR: Adjusted odds ratio; Cl: Confidence Interval; WTV: Willing to be vaccinated; non-WTV: Unwilling to be vaccinated; BMI: Body mass index; TDF: Tenofovir disoproxil fumarate; FTC: Emtricitabine.

\section{Declarations}

\section{Ethics approval and consent to participate}

The study was approved by the Ethics and Human Subjects Committee (EHSC) of Guangxi Medical University[No.20210153]. All participants were verbally informed of the purpose of the study and signed the informed consent forms. All methods were performed in accordance with the relevant guidelines and regulations.

\section{Consent for publication}

Not applicable.

\section{Availability of data and materials}

The HIV patient's datasets generated and/or analysed during the current study are not publicly available due to ethical and legal reasons, but are available from the corresponding author on request.

\section{Competing interests}

The authors declare that they have no competing interests.

\section{Funding}

This study was funded by Guangxi Bagui Scholar (to Junjun Jiang), Guangxi Medical University Training Program for Distinguished Young Scholars (to Junjun Jiang). The funding bodies had no role in the design of the study and collection, analysis, and interpretation of data and in writing the manuscript.

\section{Authors' contributions}


$\mathrm{JJ}, \mathrm{LY}$ and $\mathrm{HL}$ conceived and designed the study. JS, JJ and ZJ conducted the data analysis, literature review, and wrote the main manuscript text. RC, BL, LC and YW carried out questionnaire survey. TQ, MS and $Y L$ were involved in the study supervision, data collection, and interpretation of the data. $F Q$, $Y L$ and PP assisted with data management and data analysis. All authors reviewed the manuscript.

\section{Acknowledgements}

We would like to express our gratitude to all of staffs from the Chongzuo, Guigang, Laibing, Qinzhou, Yulin Center for Disease Prevention and Control in Guangxi, China, for their collecting and providing epidemiological data of local HIV/AIDS.

\section{Authors' information}

${ }^{1}$ Guangxi Key Laboratory of AIDS Prevention and Treatment, School of Public Health \& Life Science Institute, Guangxi Medical University, Nanning, China.

${ }^{2}$ Guangxi Key Laboratory of AIDS Prevention and Treatment, School of Public Health, Guangxi Medical University, Nanning, China.

\section{References}

1. WHO. WHO Coronavirus (COVID-19). 2021. https://covid19.who.int/. Accessed 10 Oct 2021.

2. Zhao S, Zhang H, Yang X, Zhang H, Chen Y, Zhan Y, Zhang X, Jiang R, Liu M, Liu L et al: Identification of potent human neutralizing antibodies against SARS-CoV-2 implications for development of therapeutics and prophylactics. Nature communications 2021, 12(1):4887.

3. Feng J, Li Q: How to ensure vaccine safety: An evaluation of China's vaccine regulation system. Vaccine 2021, 39(37):5285-5294.

4. Palacios R, Patino EG, de Oliveira Piorelli R, Conde M, Batista AP, Zeng G, Xin Q, Kallas EG, Flores J, Ockenhouse CF et al: Double-Blind, Randomized, Placebo-Controlled Phase III Clinical Trial to Evaluate the Efficacy and Safety of treating Healthcare Professionals with the Adsorbed COVID-19 (Inactivated) Vaccine Manufactured by Sinovac - PROFISCOV: A structured summary of a study protocol for a randomised controlled trial. Trials 2020, 21(1):853.

5. Flanagan KL, Maclntyre CR, Mclntyre PB, Nelson MR: SARS-CoV-2 Vaccines: Where Are We Now? J Allergy Clin Immunol Pract 2021.

6. Pawlowski C, Lenehan P, Puranik A, Agarwal V, Venkatakrishnan AJ, Niesen MJM, O'Horo JC, Virk A, Swift MD, Badley AD et al: FDA-authorized mRNA COVID-19 vaccines are effective per real-world evidence synthesized across a multi-state health system. Med (N Y) 2021, 2(8):979-992 e978.

7. Zhou F, Yu T, Du R, Fan G, Liu Y, Liu Z, Xiang J, Wang Y, Song B, Gu X et al: Clinical course and risk factors for mortality of adult inpatients with COVID-19 in Wuhan, China: a retrospective cohort study. Lancet 2020, 395(10229):1054-1062. 
8. Sahin I, Haymana C, Demir T, Demirci I, Tasci I, Atmaca A, Cakal E, Ata N, Emral R, Unluturk U et al: Clinical Characteristics and Outcomes of COVID-19 Patients with Overweight and Obesity: Turkish Nationwide Cohort Study (TurCObesity). Exp Clin Endocrinol Diabetes 2021.

9. UNAIDS. Confronting Inequalities-2021 Global AIDS Update. 2021.

https://www.unaids.org/sites/default/files/media_asset/2021-global-aids-update_en.pdf. Accessed 14 Jul 2021.

10. Disease Prevention and Control Administration of National Health Commission of the People's Republic of China: New progress in the prevention and treatment of AIDS in China in 2019. Chin J AIDS STD 2019, 25(12):1205.

11. Jia XC, Xia ZH, Shi N, Wang YP, Luo ZX, Yang YL, Shi XZ: The factors associated with natural disease progression from HIV to AIDS in the absence of ART, a propensity score matching analysis. Epidemiol Infect 2020, 148:e57.

12. Ho HE, Peluso MJ, Margus C, Matias Lopes JP, He C, Gaisa MM, Osorio G, Aberg JA, Mullen MP: Clinical Outcomes and Immunologic Characteristics of Coronavirus Disease 2019 in People With Human Immunodeficiency Virus. J Infect Dis 2021, 223(3):403-408.

13. Braunstein SL, Lazar R, Wahnich A, Daskalakis DC, Blackstock OJ: Coronavirus Disease 2019 (COVID-19) Infection Among People With Human Immunodeficiency Virus in New York City: A Population-Level Analysis of Linked Surveillance Data. Clin Infect Dis 2021, 72(12):e1021-e1029.

14. Jassat W, Cohen C, Tempia S, Masha M, Goldstein S, Kufa T, Murangandi P, Savulescu D, Walaza S, Bam JL et al: Risk factors for COVID-19-related in-hospital mortality in a high HIV and tuberculosis prevalence setting in South Africa: a cohort study. Lancet HIV2021, 8(9):e554-e567.

15. Ssentongo P, Heilbrunn ES, Ssentongo AE, Advani S, Chinchilli VM, Nunez JJ, Du P: Epidemiology and outcomes of COVID-19 in HIV-infected individuals: a systematic review and meta-analysis. Sci Rep 2021, 11(1):6283.

16. Qiao YC, Xu Y, Jiang DX, Wang X, Wang F, Yang J, Wei YS: Epidemiological analyses of regional and age differences of HIV/AIDS prevalence in China, 2004-2016. Int J Infect Dis 2019, 81:215-220.

17. Dong Pengyu XP, Liu Xiaoqiang, Liu Hong, He Guozhong: Analysis of Epidemic and Prevention Situation of Major Infectious Diseases in China's Southwest Border Area. Medicine and Society 2017, 30(2):45-48.

18. Wang J, Jing R, Lai X, Zhang H, Lyu Y, Knoll MD, Fang H: Acceptance of COVID-19 Vaccination during the COVID-19 Pandemic in China. Vaccines (Basel) 2020, 8(3).

19. Lin Y, Hu Z, Zhao Q, Alias H, Danaee M, Wong LP: Understanding COVID-19 vaccine demand and hesitancy: A nationwide online survey in China. PLoS Negl Trop Dis 2020, 14(12):e0008961.

20. Lazarus JV, Ratzan SC, Palayew A, Gostin LO, Larson HJ, Rabin K, Kimball S, El-Mohandes A: A global survey of potential acceptance of a COVID-19 vaccine. Nat Med 2021, 27(2):225-228.

21. Tesoriero JM, Swain CE, Pierce JL, Zamboni L, Wu M, Holtgrave DR, Gonzalez CJ, Udo T, Morne JE, Hart-Malloy R et al: COVID-19 Outcomes Among Persons Living With or Without Diagnosed HIV Infection in New York State. JAMA Netw Open 2021, 4(2):e2037069. 
22. Jewell BL, Mudimu E, Stover J, Ten Brink D, Phillips AN, Smith JA, Martin-Hughes R, Teng Y, Glaubius $\mathrm{R}$, Mahiane SG et al: Potential effects of disruption to HIV programmes in sub-Saharan Africa caused by COVID-19: results from multiple mathematical models. Lancet HIV2020, 7(9):e629-e640.

23. Chilot D, Woldeamanuel Y, Manyazewal T: COVID-19 Burden on HIV Patients Attending Antiretroviral Therapy in Addis Ababa, Ethiopia: A Multicenter Cross-Sectional Study. Res Sq 2021.

24. WHO. The cost of inaction: COVID-19-related service disruptions could cause hundreds of thousands of extra deaths from HIV.2020. https://www.who.int/news/item/11-05-2020-the-cost-of-inactioncovid-19-related-service-disruptions-could-cause-hundreds-of-thousands-of-extra-deaths-from-hiv. Accessed 2 Jun 2020.

25. Yang T, Liu B, Yue L, Xie T, Li H, Shao M, Yang R, Luo F, Long R, Xie Z: Preclinical safety assessment of a combined vaccine against Hepatitis a virus and enterovirus 71. Vaccine 2021, 39(29):39523963.

26. Sullender WM, Fowler KB, Gupta V, Krishnan A, Ram Purakayastha D, Srungaram VIn R, Lafond KE, Saha S, Palomeque FS, Gargiullo $P$ et al: Efficacy of inactivated trivalent influenza vaccine in rural India: a 3-year cluster-randomised controlled trial. Lancet Glob Health 2019, 7(7):e940-e950.

27. Jara A, Undurraga EA, Gonzalez C, Paredes F, Fontecilla T, Jara G, Pizarro A, Acevedo J, Leo K, Leon F et al: Effectiveness of an Inactivated SARS-CoV-2 Vaccine in Chile. N Engl J Med 2021, 385(10):875884.

28. Hu Y, Chen S: What can we learn from COVID-19 vaccine R\&D in China? A discussion from a public policy perspective. J Travel Med 2021, 28(4).

29. Dodd RH, Pickles K, Cvejic E, Cornell S, Isautier JMJ, Copp T, Nickel B, Bonner C, Batcup C, Muscat DM et al: Perceived public health threat a key factor for willingness to get the COVID-19 vaccine in Australia. Vaccine 2021.

30. Acharya SR, Moon DH, Shin YC: Assessing Attitude Toward COVID-19 Vaccination in South Korea. Front Psychol 2021, 12:694151.

31. Dagan N, Barda N, Kepten E, Miron O, Perchik S, Katz MA, Hernan MA, Lipsitch M, Reis B, Balicer RD: BNT162b2 mRNA Covid-19 Vaccine in a Nationwide Mass Vaccination Setting. N Engl J Med 2021, 384(15):1412-1423.

32. WHO. The-race-for-a-covid-19-vaccine-explained. 2021. https://www.who.int/zh/news-room/featurestories/detail/the-race-for-a-covid-19-vaccine-explained. Accessed 12 Jan 2021.

33. LI Zheng, LIU Ye, LI Chun-yang: Application and progress in research of live attenuated vaccines. Chin J Biologicals 2018, 31(2):205-208.

34. Shekatkar M, Kheur S, Gupta AA, Arora A, Raj AT, Patil S, Khan SS, Desai A, Carroll WB, Awan KH: Oral candidiasis in human immunodeficiency virus-infected patients under highly active antiretroviral therapy. Dis Mon 2021, 67(9):101169.

35. del Amo J, Polo R, Moreno S, Diaz A, Martinez E, Arribas JR, Jarrin I, Hernan MA, Collaboration SHC-: Incidence and Severity of COVID-19 in HIV-Positive Persons Receiving Antiretroviral Therapy A Cohort Study. Ann Intern Med 2020, 173(7):536-+.

Page $13 / 14$ 
36. Boulle A, Davies MA, Hussey H, Ismail M, Morden E, Vundle Z, Zweigenthal V, Mahomed H, Paleker M, Pienaar $\mathrm{D}$ et al: Risk factors for COVID-19 death in a population cohort study from the Western Cape Province, South Africa. Clin Infect Dis 2020.

37. Di Biagio A, Ricci E, Calza L, Squillace N, Menzaghi B, Rusconi S, Orofino G, Bargiacchi O, Molteni C, Valsecchi $L$ et al: Factors associated with hospital admission for COVID-19 in HIV patients. AIDS 2020, 34(13):1983-1985.

38. Inciarte A, Gonzalez-Cordon A, Rojas J, Torres B, de Lazzari E, de la Mora L, Martinez-Rebollar M, Laguno M, Callau P, Gonzalez-Navarro A et al: Clinical characteristics, risk factors, and incidence of symptomatic coronavirus disease 2019 in a large cohort of adults living with HIV: a single-center, prospective observational study. AIDS 2020, 34(12):1775-1780.

39. HIV/AIDS and hepatitis C Group, Society of Infectious Diseases, CMA: Expert recommendation for novel severe acute respiratory syndrome coronavirus 2 (SARS CoV 2) vaccination in patients with HIV infection. Chin J Intern Med 2021, 60(7):615-618.

40. Lazarus JV, Wyka K, Rauh L, Rabin K, Ratzan S, Gostin LO, Larson HJ, El-Mohandes A: Hesitant or Not? The Association of Age, Gender, and Education with Potential Acceptance of a COVID-19 Vaccine: A Country-level Analysis. J Health Commun 2020, 25(10):799-807.

41. Zhou XJ, Zhu QY, Li JJ, Lan GH, Liang SS, Liu SF, Liu XH, Meng Q, Zhou CX, Shen ZY: Characteristics of HIV-infected persons without long term disease progress and related factors in Guangxi Zhuang Autonomous Region. Zhonghua Liu Xing Bing Xue Za Zhi 2019, 40(1):70-73.

42. Wu JT, Leung K, Leung GM: Nowcasting and forecasting the potential domestic and international spread of the 2019-nCoV outbreak originating in Wuhan, China: a modelling study. Lancet 2020, 395(10225):689-697.

\section{Tables}

Due to technical limitations, tables are only available as a download in the Supplemental Files section.

\section{Supplementary Files}

This is a list of supplementary files associated with this preprint. Click to download.

- Tablesnew.pdf

- Supplementarytablesnew.pdf 